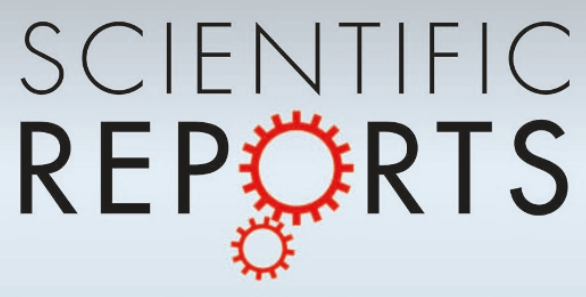

OPEN

SUBJECT AREAS:

SOLID-STATE CHEMISTRY

METAL-ORGANIC FRAMEWORKS

Received

9 July 2013

Accepted

8 August 2013

Published

6 September 2013

Correspondence and requests for materials should be addressed to

T.B. (bein@lmu.de)

* These authors contributed equally to this work.

\section{Highly sensitive and selective fluoride detection in water through fluorophore release from a metal-organic framework}

Florian M. Hinterholzinger ${ }^{1 *}$, Bastian Rühle ${ }^{1 *}$, Stefan Wuttke ${ }^{1}$, Konstantin Karaghiosoff ${ }^{2} \&$ Thomas Bein $^{1}$

'Department of Chemistry and Center for NanoScience (CeNS), University of Munich (LMU), Butenandtstraße 11 (E), 81377 München (Germany), ${ }^{2}$ Department of Chemistry, University of Munich (LMU), Butenandtstraße 5-13 (D), 81377 München (Germany).

The detection, differentiation and visualization of compounds such as gases, liquids or ions are key challenges for the design of selective optical chemosensors. Optical chemical sensors employ a transduction mechanism that converts a specific analyte recognition event into an optical signal. Here we report a novel concept for fluoride ion sensing where a porous crystalline framework serves as a host for a fluorescent reporter molecule. The detection is based on the decomposition of the host scaffold which induces the release of the fluorescent dye molecule. Specifically, the hybrid composite of the metal-organic framework $\mathrm{NH}_{2}$-MIL-101(Al) and fluorescein acting as reporter shows an exceptional turn-on fluorescence in aqueous fluoride-containing solutions. Using this novel strategy, the optical detection of fluoride is extremely sensitive and highly selective in the presence of many other anions.

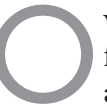

ver the past decades a great deal of attention has been devoted to the discovery of new analytical methods for the reliable detection of target species ${ }^{1-4}$. The real-time monitoring of fluoride ion concentration in aqueous and physiological media as well as its quantitative determination across a large concentration range is of vital importance for various environmental and health-care issues as well as in industrial and scientific application $s^{5-8}$. Several strategies have been reported on fluoride detection using recognition-transduction schemes based on the optical read-out of a luminescent or colorimetric signal. Examples include molecular recognition protocols using boron ${ }^{5,6,9-11}$ or stibonium ${ }^{12}$-based anion receptors, or mesoporous silica host materi$\mathrm{als}^{13}$ that are equipped with a reporter molecule to monitor either luminescence quenching or turn-on processes, whereas recently a sensitive colorimetric fluoride sensor based on supramolecular interactions such as charge or electron transfer, respectively, was described ${ }^{14}$. However, most of the molecule-based analytical methods suffer from interference with other anions or are incompatible with aqueous media, which greatly limits the scope of their use $\mathrm{e}^{15,16}$. Here we demonstrate how the analyte-induced degradation of a hybrid host material with embedded reporter molecules can be efficiently used as an extremely sensitive signal transduction mode (Fig. 1). It is thus anticipated that our new recognition-transduction mechanism contributes to the development of optically encoded chemosensors and hence for the sensitive visualization of specific host-guest interactions. Metal-organic frameworks (MOFs), also known as porous coordination polymers $(\mathrm{PCPs})^{17-19}$, have attracted great interest in the last decade as a new class of highly porous materials ideally suited for applications in gas adsorption, separation processes $^{20-22}$ and chemical sensing ${ }^{23-25}$. The underlying modular concept of combining inorganic metal-clusters and organic linker molecules allows for the targeted design of three-dimensional crystalline molecular skeletons with defined pore dimensions, uniform pore shapes and versatile functionalities.

Our strategy for visualizing the recognition of a target ion requires the implementation of a fluorescent reporter molecule into the confined crystalline environment of a hybrid host material. As a consequence of the confinement of the reporter molecules within the three-dimensional host, almost complete fluorescence quenching is observed. However, the selective trapping of fluoride ions from aqueous analytes directly correlates with an intense turn-on fluorescence signal that can easily be monitored by fluorescence spectroscopy. More specifically, the turn-on of fluorescence that is triggered by the controlled decomposition of the host material exhibits great advantages over luminescence quenching methods reported for luminescent $\mathrm{MOFs}^{23,26}$. The turn-on fluorescence is an extremely sensitive technique, having the capability to detect even single molecules in solution and has great potential for the highly sensitive monitoring of the fluoride ion concentration in different media. Although there are several reports dealing with the intrinsic luminescence of MOFs and its use in chemical sensing ${ }^{2,3,27,28}$, none of 


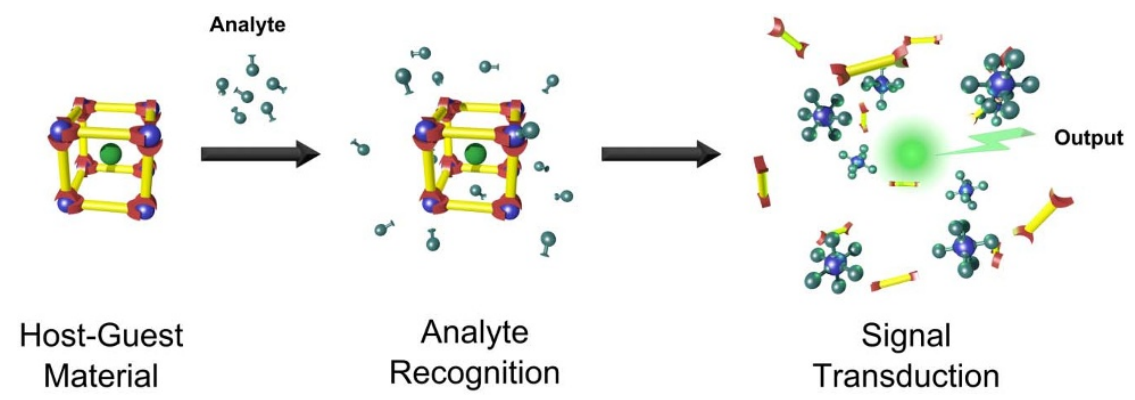

Figure 1 Schematic illustration of the recognition-transduction scheme by metal-anion complexation-induced decomposition of a MOF framework. The MOF scaffold with covalently entrapped reporter molecules serves as a non-emissive host-guest material for the detection of analyte species. The recognition is realized as a competitive metal-anion complexation in an aqueous analyte-containing environment. The degradation process leads directly to the turn-on of the fluorescence of the reporter molecules, which is correlated with the fluoride ion concentration.

these optical detection schemes tolerates aqueous environments and simultaneously shows turn-on fluorescence response triggered by the highly selective capture of fluoride ions.

Here we report on highly selective and extremely sensitive fluoride sensor based on an amino-functionalized metal-organic framework designated $\mathrm{NH}_{2}-\mathrm{MIL}-101(\mathrm{Al})^{29}$ that covalently binds fluorescein 5(6)-isothiocyanate (FITC) as fluorescent reporter molecule. We demonstrate an unprecedented methodology for the detection of fluoride ions in aqueous solutions that is based on the decomposition of the MOF and provides an intense turn-on switching response induced by the release of the quenched reporter molecules. The intact crystalline MOF material with embedded fluorescein dyes itself is non-fluorescent and selectively binds fluoride ions from water. The capture of fluoride ions results in the formation of different aluminum complexes, which induces the structural decomposition of the MOF host. The detection mechanism after infiltrating the MOF with an aqueous fluoride-containing analyte solution is revealed by ${ }^{19} \mathrm{~F}$ and ${ }^{27} \mathrm{Al}-\mathrm{NMR}$ titration experiments. To the best of our knowledge, the fluorescein-labeled MOF composite material is the first example of a MOF-based fluoride sensor system which tolerates aqueous environments, exhibits high selectivity versus other anions and covers a broad range of anion concentrations. We anticipate that the general concept of creating a fluorescence output by the controlled release of quenched fluorescence dye molecules from a porous host can be extended to other analyte detection schemes, thus adding to the toolbox for the development of advanced optical chemosensors and the understanding of signal transduction schemes.

\section{Results}

Recently, the group of F. Kapteijn reported on the amino-functionalized analogue of a large-pore metal-organic framework, designated $\mathrm{NH}_{2}$-MIL-101(Al) ${ }^{29}$. The aluminum-based MOF scaffold with implemented amino-functionality represents an ideal candidate for the post-synthetic dye-labeling that was performed by exposing the porous host to FITC at room temperature. Powder X-ray diffraction (PXRD) measurements of the as-synthesized MOF material, as well as the post-synthetically fluorescein-labeled host are in good agreement with simulated data indicating that the crystalline structure remains intact upon dye-labeling (Supplementary Fig. S1). Scanning electron microscope measurements (SEM) additionally confirm that the crystal morphology of the host MOF does not change significantly after being post-synthetically modified with fluorescein dye molecules (Supplementary Fig. S2). After dye loading, a significant decrease of the specific surface area from $2315 \mathrm{~m}^{2} / \mathrm{g}$ to $698 \mathrm{~m}^{2} / \mathrm{g}$ as well as a reduction of the pore volume of the host material is revealed by physisorption experiments. This indicates a successful introduction of FITC into the porous structure of the host (Supplementary Fig. S3). Infrared and Raman spectroscopy show the presence of a strong characteristic absorption band corresponding to the isothiocyanate group of free FITC, whereas no such absorption band is observed after fluorescein is incorporated into the host framework (Supplementary Fig. S6 + S7). Fluorescence spectroscopy shows that the fluorescence intensity of the fluorescein guests drops significantly (almost to the background level, see Fig. 2) once they are incorporated into the framework.

Fluorescence titrations of $\mathrm{NH}_{2}$-MIL-101(Al)-FITC with sodium salt solutions of different anions were used to demonstrate the selectivity of the sensor (see Fig. 3). The MOF-based fluoride sensor shows very high selectivity against many other ions in aqueous solution.

Additionally, more detailed information about the different fluoride species in solution as well as mechanistic insights into the dissolution process were gained by performing NMR titration experiments, which prove that the framework is indeed dissolved by coordination of fluoride to aluminum ions from the framework.

The sensitivity of $\mathrm{NH}_{2}$-MIL-101(Al)-FITC towards fluoride was investigated by fluorescence titrations experiments (see Fig. 4). The data demonstrate a very high sensitivity over a broad range of fluoride ion concentrations.

\section{Discussion}

We believe that the significant drop of fluorescence intensity after incorporation of the dye into the host scaffold (see Fig. 2) is caused by the well-known concentration- or self-quenching-effect of fluorescent dyes like fluorescein ${ }^{30,31}$, or by interactions of fluorescein either with aromatic groups of the linker molecules, the metal centers, or the amino groups inside the MOF. Inspired by this observation, we developed the novel MOF-based fluoride sensing concept using the selective release of the fluorescent dye: Since the framework is not connected by strong covalent bonds, it can be broken up under mild conditions by other, more strongly coordinating ligands for the respective metal. While this "instability" can certainly be a disadvantage in certain applications, it is of key importance for the working principle of the fluoride sensor and the new sensing pathway presented in this work: upon replacing the MOF-linker molecules by other ligands, the fluorescent guest is released into the solution where its fluorescence is dequenched and a strong increase in the fluorescence signal can be detected. While the degradation of the framework renders this pathway non-reversible, we do not believe this to be a major setback, since all the reagents for the material used in this work are readily commercially available, the synthesis of the material is relatively fast and easy, and only very small amounts of the material are needed for performing the analysis. Due to the fact that the degradation of the framework is based on different affinities of the metal ions constituting the MOF towards linker and analyte molecules, this pathway has the potential of being highly selective and tailorable by choosing suitable MOFs (i.e. metal/linker combinations) for the respective target analytes. The fact that the release of the fluorescent dye results in a fluorescence increase rather than a quenching or loss of color contributes to the high sensitivity of this approach. Both the high selectivity based on strong interactions of 
a

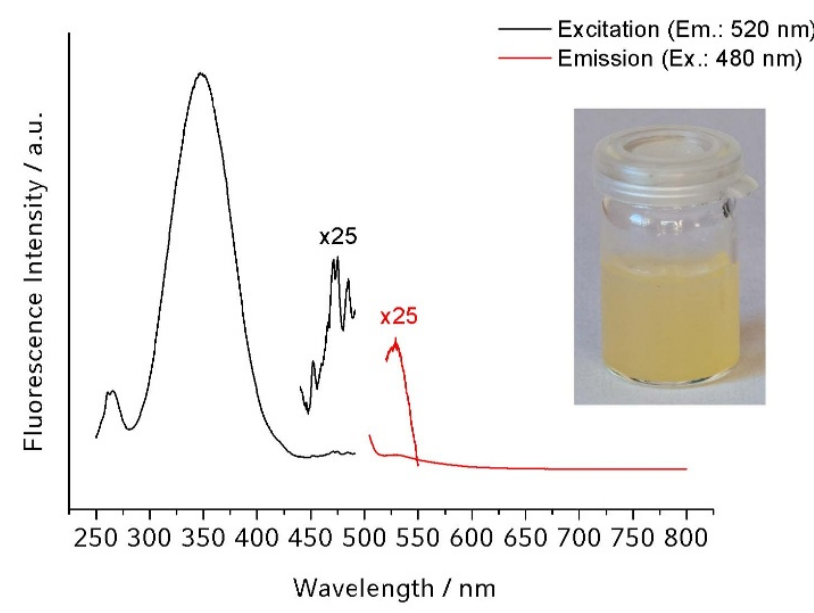

C

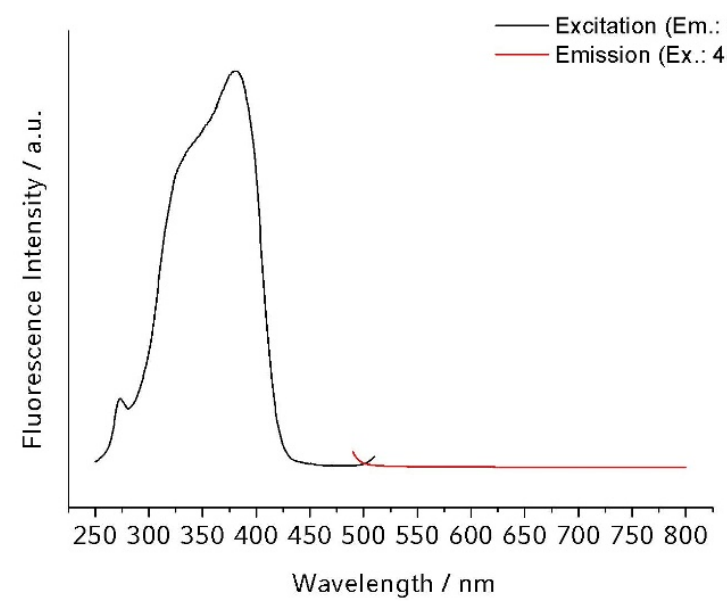

b

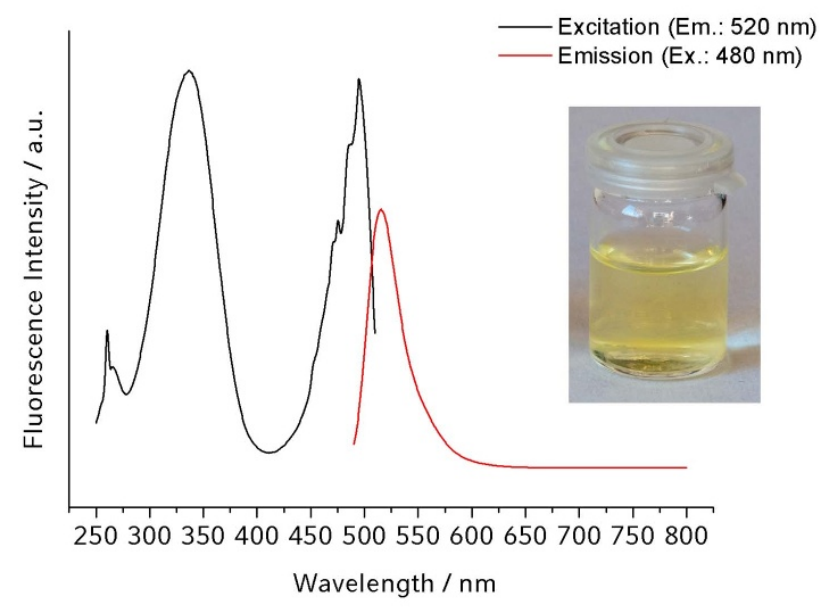

d

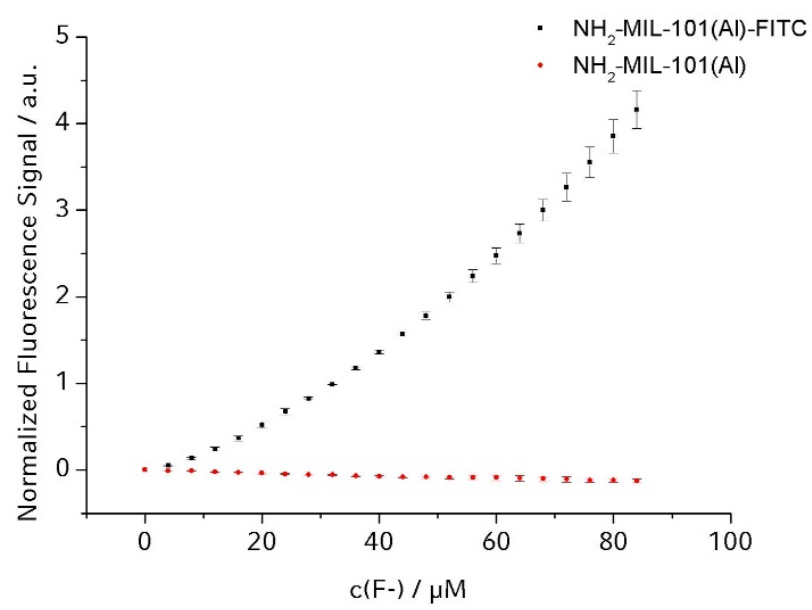

Figure $2 \mid$ Fluorescence spectroscopy data. (a), Excitation and emission spectra of $\mathrm{NH}_{2}$-MIL-101(Al)-FITC before fluoride addition. (b), Excitation and emission spectra of $\mathrm{NH}_{2}$-MIL-101(Al)-FITC after fluoride addition. (c), Excitation and emission spectra of 2-amino terephthalic acid, representing the linker molecule in $\mathrm{NH}_{2}-\mathrm{MIL}-101(\mathrm{Al})$. (d), Comparison of fluorescence titrations of $\mathrm{NH}_{2}-\mathrm{MIL}-101(\mathrm{Al})$-FITC and unlabeled NH${ }_{2}-\mathrm{MIL}-101(\mathrm{Al})$ (error bars indicate the deviation of two individual measurements with different MOF and titration stock solutions; for a more detailed description of experimental details, data analysis, and raw spectral data see Supplementary Information). Insets in (a) and (b), Photographs of the MOF stock solution before (a) and after (b) fluoride addition.

aluminum with fluoride ions ${ }^{32}$ and the high sensitivity based on the fluorescence dequenching are discussed in the following.

The remarkable selectivity of the sensor is demonstrated by fluorescence titrations of $\mathrm{NH}_{2}$-MIL-101(Al)-FITC with sodium salt solutions of different anions. The anions that were examined for interference in this work were chosen to reflect species that are typically present in natural water and that are also usually investigated when assessing the performance of a fluoride detection method $^{12-14,33}$. Our investigations show that there is virtually no interference by other halides (see Fig. 3). The strongest interference among the anions tested in this work is caused by sodium bicarbonate, which can be expected to mimic the coordination chemistry of the linker molecules, i.e. coordination of aluminum via carboxylate groups. Interestingly, sodium acetate shows only very weak interference, although it also exhibits a carboxylate group. The absence of strong acetate interference also proves that the recorded fluorescence increase does not simply depend on an increase of the $\mathrm{pH}$, since the basicity of acetate is higher than that of fluoride $\left(\mathrm{pK}_{\mathrm{A}}\left(\mathrm{CH}_{3} \mathrm{COOH}\right) \approx\right.$ 4.75 vs. $\left.\mathrm{pK}_{\mathrm{A}}(\mathrm{HF}) \approx 3.45\right)^{34}$. However, the fluorescence signal is much stronger after fluoride addition than after acetate addition, indicating that the fluorescence increase is triggered by coordination of fluoride to aluminum metal centers rather than by a rise of the $\mathrm{pH}$.
Interference by other ions is a well-known issue, also for the commonly used optical fluoride detection methods in aqueous solution, such as the SPADNS method or the complexone method ${ }^{35,36}$. Hence, a process called "Bellack Distillation" 33 is usually recommended prior to sample analysis if appreciable amounts of interfering ions are present $t^{33,35,36}$. This process was shown to remove most interfering ions from the analyte solution ${ }^{33}$.

The fact that fluoride leads to a controlled decomposition of the framework is also backed up by NMR titration experiments which clearly show that differently coordinated molecular aluminum fluoride species are formed upon fluoride addition to a dispersion of the MOF in water (see Fig. 5). Fig. 5.a shows the ${ }^{19} \mathrm{~F}$ NMR spectra after sequential additions of $\mathrm{NaF}$ to $\mathrm{NH}_{2}$-MIL-101(Al)-FITC in water. The data demonstrate how the number of fluoride ions binding to aluminum increases with increasing fluoride concentration. The peaks of the aluminum complexes with high fluoride content (i.e. more than four fluoride atoms) cannot be observed here, most probably because they are too broad. Similar observations were made by Sur et al. ${ }^{37}$ and by Bodor et al. ${ }^{38}$. Sur et al. attribute the line broadening mainly to a two-site exchange between free fluoride ions and fluoroaluminum complexes, although scalar relaxation mechanisms are also discussed. Bodor et al. also attribute the broadening to 
a

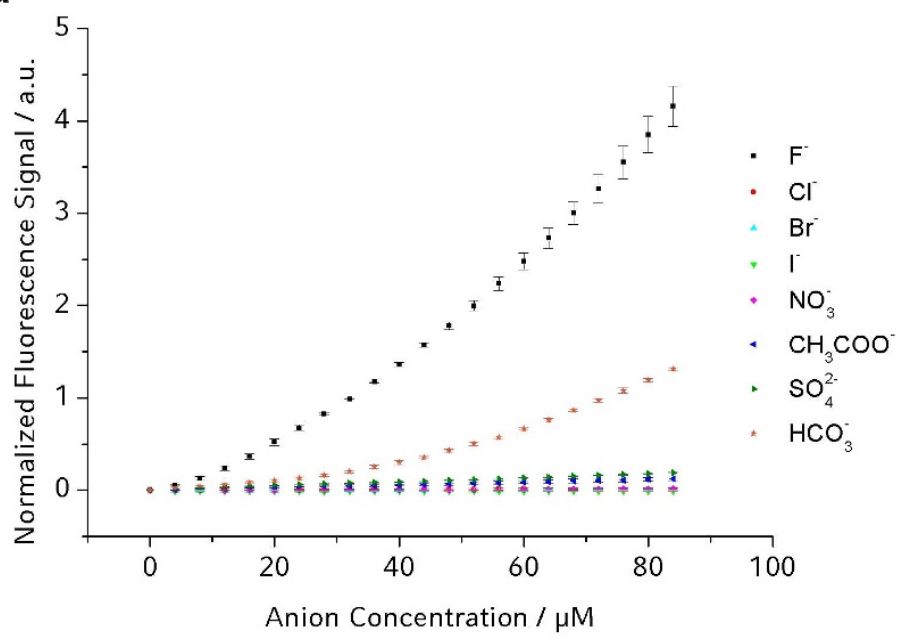

b

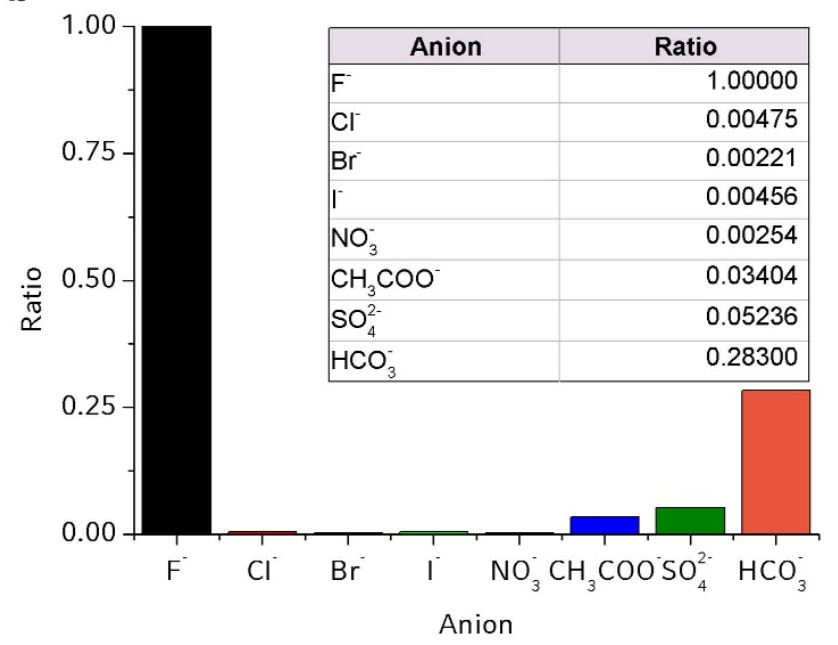

Figure 3 Sensor selectivity data obtained from fluorescence titrations with different salt solutions. (a), Data obtained from fluorescence titrations of $\mathrm{NH}_{2}$-MIL-101(Al)-FITC with sodium salts of different anions. Error bars indicate the deviation of two individual measurements with different MOF and titration stock solutions. (b), Ratio of slopes obtained by fitting a line through the origin to the data in (a). For a more detailed description of experimental details, data analysis, and raw spectral data see Supplementary Information.

fluoride exchange; however, the authors also briefly mention precipitation and the formation of mixed aluminum hydroxo species as possible causes.

Going deeper into the exact nature of the aluminum fluoride species that are formed is beyond the scope of this work, but our NMR titration experiments clearly show that differently coordinated molecular aluminum fluoride species are formed upon fluoride addition to a dispersion of the MOF in water. In agreement with the literature, we found complexes with one and two fluoride atoms bound to aluminum at low fluoride concentrations. With increasing fluoride concentration, aluminum species that are coordinated by more and more fluoride ions are formed, while the amount of aluminum species with lower fluoride amounts decreases. Interestingly, the amount of "free" fluoride (i.e. fluoride ions that are not coordinating a metal ion) observed at $\delta \approx-119.2 \mathrm{ppm}$ is very low at the beginning, and after the corresponding peak starts to develop at about $1000 \mu \mathrm{M}$, it stays at a low level. We propose the following detection mechanism: aluminum fluoride complexes and linker molecules (together with the fluorescent dye) are liberated from the framework into the solution via a displacement of linker molecules by fluoride ions. This is also backed up by the ${ }^{27} \mathrm{Al} \mathrm{NMR}$ spectra (see Fig. 5.b), which feature a broad peak at $\delta \approx 4$ ppm that can be assigned to $\left[\mathrm{AlF}_{\mathrm{x}}\left(\mathrm{H}_{2} \mathrm{O}\right)_{6-\mathrm{x}}\right]^{(3-\mathrm{x})+37,38}$. Before fluoride addition, no signals can be observed in the ${ }^{27} \mathrm{Al} \mathrm{NMR}$, indicating that there is no appreciable amount of aluminum present in the solution at the beginning. Only after addition of fluoride, aluminum fluoride complexes can be found in solution. This indicates that the aluminum source is really the MOF and that the fluoride complexes that can be observed by NMR are indeed formed by a degradation of the metalorganic framework.

In addition to the remarkable selectivity of $\mathrm{NH}_{2}$-MIL-101(Al)FITC towards fluoride, we could also demonstrate a very high sensitivity covering a broad range of fluoride ion concentrations from approximately $15 \mu \mathrm{g}$ up to at least $1600 \mu \mathrm{g}$ of fluoride per $1 \mathrm{~L}$ of water, or 15 to $1600 \mathrm{ppb}$. (see Fig. 4).

We believe that the main reason for the high sensitivity is the efficient quenching and dequenching of fluorescein fluorescence upon incorporation into and liberation from the host framework, respectively. The slight deviation from linear behavior at low fluoride concentration (which can be better seen in a non-double-logarithmic plot, as for example in Fig. 1.d) is attributed to the initial coordination of fluoride ions to trace amounts of free aluminum precursor still present in the porous framework or from coordination to unsaturated metal centers inside the MOF. Both effects do not lead to a replacement of linker molecules by fluoride and hence the amount of fluorescein that is released into the solution is lower than expected. Once all these metal ions are coordinated by fluoride, further addition of fluoride results in linker displacement and liberation of fluorescein into the solution and the concomitant fluorescence dequenching discussed above. Assuming a homogeneous distribution of fluorescein throughout the framework, the fluorescence signal should hence increase linearly with fluoride concentration, until the framework is more or less completely dissolved, at which point the fluorescence signal should level off. However, that point was not reached in the titrations shown here.

In this study we have introduced a new signal transduction scheme in the field of MOFs, which is based on the efficient quenching of fluorescent guests upon incorporation into the porous framework

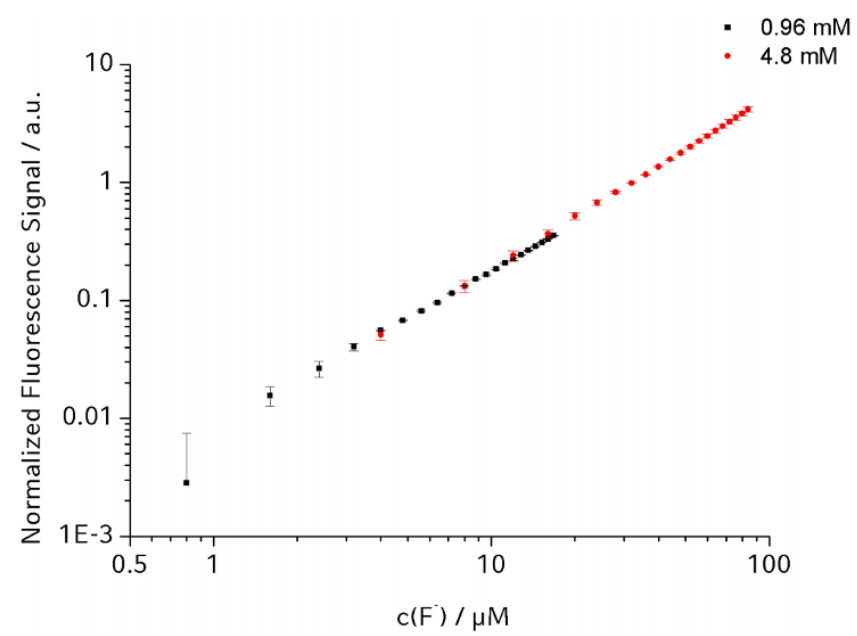

Figure $4 \mid$ Fluorescence titration curves. The data show fluorescence titrations of $\mathrm{NH}_{2}$-MIL-101(Al)-FITC with two titration solutions of different concentration. Error bars indicate the deviation of two individual measurements with different MOF and titration stock solutions. For a more detailed description of experimental details, data analysis, and raw spectral data see Supplementary Information. 

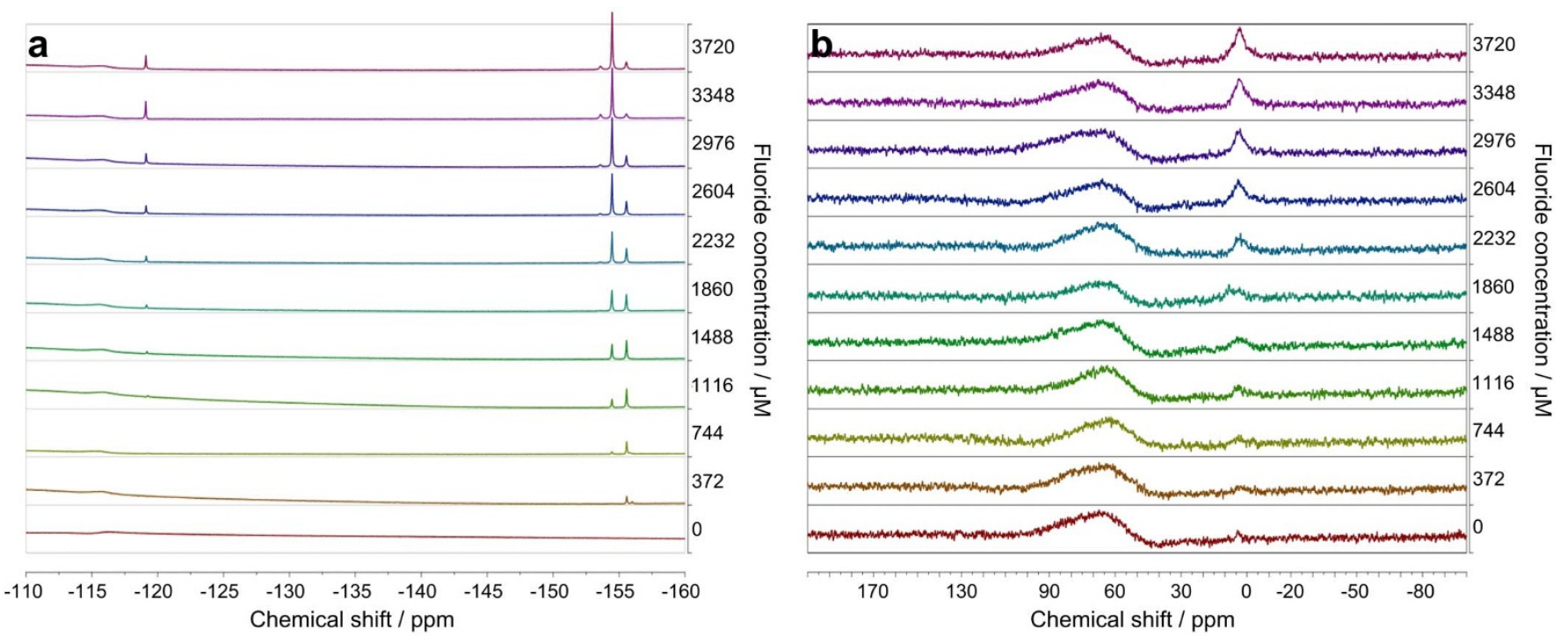

Figure $5 \mid{ }^{19} \mathrm{~F}$ and ${ }^{27} \mathrm{Al}$ NMR titration of $\mathrm{NH}_{2}$-MIL-101(Al)-FITC. (a), ${ }^{19} \mathrm{~F}$ NMR. (b), ${ }^{27} \mathrm{Al} \mathrm{NMR}$. The broad signal in the ${ }^{27} \mathrm{Al} \mathrm{NMR}$ between $\delta \approx 50$ ppm and $\delta \approx 110 \mathrm{ppm}$ arises from aluminum atoms from the NMR probe head or the NMR glass tube (see Supplementary Information). The concentration of fluoride was adjusted to the values given in the figure. For peak assignments see Table 1. For a detailed description of experimental parameters, FID processing and raw spectral data see Supplementary Information.

and the subsequent dequenching upon analyte-specific degradation of the host material. This new sensing concept was illustrated with the dye-loaded MOF $\mathrm{NH}_{2}$-MIL-101(Al), thus introducing a highly sensitive and selective fluoride sensor that is compatible with aqueous analyte solutions. Deeper insights into the transduction mechanism were gained by ${ }^{19} \mathrm{~F}$ and ${ }^{27} \mathrm{Al}$ NMR titration studies, revealing that the porous host framework is indeed dissolved upon fluoride ion addition due to competing coordination of fluoride to metal centers inside the metal-organic framework. We believe that the high sensitivity of the system is controlled by the coordination chemistry and the different affinities of the metal ions inside the framework towards linker molecules, fluoride ions, and other interfering ions. Fluorescence titration experiments were used to quantitatively show the remarkable selectivity and sensitivity of our fluoride sensor. We attribute the high sensitivity to the fact that a turn-on fluorescence signal rather than a color- or fluorescence-quenching based signal transduction scheme is used. All in all, the sensitivity of the sensor presented in this work is far better than that of the commonly used optical fluoride detection methods in aqueous solution according to data presented by the World Health Organization (WHO) and the American Public Health Association ${ }^{35,36}$, namely the sodium-2(parasulfophenylazo)-dihydroxy-3,6-napthalene disulfonate (SPADNS) method and the complexone method. These references give a fluoride detection range of 100 to $1400 \mathrm{ppb}$ for the SPADNS method and a range of 50 to $2000 \mathrm{ppb}$ for the complexone method, at comparable anion selectivity. In our work, we have demonstrated a detection range from 15 up to at least $1600 \mathrm{ppb}$. The sensitivity is also comparable to other optical fluoride detection methods recently discussed in the literature, which can detect fluoride concentrations in the low $\mathrm{ppm}^{11,12}$ or also in the $\mathrm{ppb}^{10,13,14}$ range. However, these methods typically require a solvent mixture of water and an organic solvent such as chloroform ${ }^{10}$, methanol ${ }^{11}$, acetonitrile ${ }^{13}$ or $\mathrm{DMSO}^{12,14}$, or need to be carried out in a buffer at acidic $\mathrm{pH}^{11,12,13}$ or in the presence of other compounds such as $\mathrm{CTAB}^{12}$. Moreover, in some cases the use of organic solvent mixtures made it necessary to use tetraalkylammonium salts instead of the naturally more abundant and relevant sodium or potassium salts when studying the selectivity and sensitivity ${ }^{10,14}$. In contrast, the method reported here works at neutral $\mathrm{pH}$ and without the need for organic solvents or other additives.

We believe that the general concept of releasing fluorescent guest molecules from MOFs can be extended to other combinations of analytes and metal coordination environments, thus adding to the growing toolbox of optical chemosensors.

\section{Methods}

Synthesis of $\mathrm{NH}_{2}$-MIL-101(Al) and $\mathrm{NH}_{2}$-MIL-101(Al)-FITC. The solvotherma synthesis of $\mathrm{NH}_{2}-\mathrm{MIL}-101(\mathrm{Al})$ was carried out according to a slightly modified literature synthesis ${ }^{28}$. In a $50 \mathrm{~mL}$ glass reactor, aluminum(III) chloride hexahydrate $(0.51 \mathrm{~g}, 2.11 \mathrm{mmol}, 1.0 \mathrm{eq})$ and 2 -amino terephthalic acid $(0.56 \mathrm{~g}, 3.09 \mathrm{mmol}$, 1.5 eq.) were dissolved in $30 \mathrm{~mL} \mathrm{~N}, N$-dimethylformamide (DMF) in an ultrasonic bath. The sealed glass reactor was kept for $72 \mathrm{~h}$ in a preheated oven at $403 \mathrm{~K}$. The resulting yellow powder was filtered under vacuum and washed with acetone. To remove organic species trapped within the pores, the samples were extracted in boiling methanol for $8 \mathrm{~h}$ and stored at $373 \mathrm{~K}$.

For dye loading experiments, fluorescein $5(6)$-isothiocyanate $(15.0 \mathrm{mg}$, $0.0385 \mathrm{mmol})$ was dissolved in absolute ethanol $(50 \mathrm{~mL}) . \mathrm{NH}_{2}-\mathrm{MIL}-101(\mathrm{Al})$ (100 mg, $0.157 \mathrm{mmol}$ ) was added to the ethanolic dye solution and left for $48 \mathrm{~h}$ on an orbital shaker at room temperature. $\mathrm{NH}_{2}-\mathrm{MIL}-101(\mathrm{Al})$-FITC was filtered and repeatedly washed with ethanol and deionized water.

Characterization. Powder X-ray diffraction (XRD) measurements were performed using a Bruker D8 diffractometer $\left(\mathrm{Cu}-\mathrm{K}_{\alpha 1}=1.5406 \AA ; \mathrm{Cu}-\mathrm{K}_{\alpha 2}=1.5444 \AA\right)$ in theta-theta geometry equipped with a Lynx-Eye detector $\left(2 \theta=2-45^{\circ}\right.$; $\left.\Delta 2 \theta=0.05^{\circ}\right)$. Nitrogen physisorption measurements were performed on a Quantachrome Instruments Autosorb at $77 \mathrm{~K}$ after outgassing for 12 hours at $393 \mathrm{~K}$. Thermogravimetric analyses of the bulk samples were performed on a Netzsch STA $440 \mathrm{C} \mathrm{TG} / \mathrm{DSC}$ with a heating rate of $1 \mathrm{~K} \mathrm{~min}^{-1}$ in a stream of synthetic air at about $25 \mathrm{~mL} \mathrm{~min}^{-1}$. Scanning electron microscopy (SEM) images were recorded with a JEOL JSM-6500F microscope equipped with a field emission gun. Fluorescence titrations were carried out using a Hamilton Microlab 500 semiautomatic precision liquid processor connected to a PTI fluorescence spectrometer. Dynamic light scattering (DLS) was performed on a Malvern Zetasizer-Nano instrument. NMR spectra were recorded on a Jeol EX 400 or a Jeol Eclipse 400 instrument at room temperature and at a frequency of $376.17 \mathrm{MHz}$ with 2048 scans for ${ }^{19} \mathrm{~F}$ NMR and 104.17 MHz with 4096 scans for ${ }^{27} \mathrm{Al} \mathrm{NMR}$.

Fluorescence titrations of $\mathrm{NH}_{2}-\mathrm{MIL}-101(\mathrm{Al})$-FITC. For fluorescence titration measurements, a stock solution of $\mathrm{NH}_{2}$-MIL-101(Al)-FITC in water with a mass concentration of $1 \mathrm{mg} / \mathrm{mL}$ was freshly prepared and sonicated very briefly (a few seconds) in an ultrasonic bath to obtain a homogeneous suspension of the MOF in water with a hydrodynamic particle size of approximately $300 \mathrm{~nm}$ as determined by dynamic light scattering (see Fig. S10). Then, $100 \mu \mathrm{L}$ of this stock solution was diluted in $2900 \mu \mathrm{L}$ of water (resulting in a total volume of $3 \mathrm{~mL}$ and a final mass concentration of $33 \mu \mathrm{g} / \mathrm{mL}$ ) in a quartz glass cuvette and stirred magnetically at room temperature. While stirring, the fluorescence emission was monitored continuously at $520 \mathrm{~nm}$ every second, using an excitation wavelength of $485 \mathrm{~nm}$ and an integration time of 1 second. The bandpass for excitation and emission was $4 \mathrm{~nm}$. When the solution was equilibrated and the fluorescence signal did not change any more over time (typically after 20-40 minutes), a sodium fluoride solution (freshly prepared by dissolving an appropriate amount of $\mathrm{NaF}$ in water) was dispensed stepwise in 
Table 1 | Chemical shifts observed during the NMR titration experiments shown in Figure 5. The peak assignments are based on references ${ }^{37,38}$

Chemical Shift [ppm]

Fluoride Species

Chemical Shift [ppm]

Aluminum Species

$-119.2$

$-153.6$

"Free" $\mathrm{F}^{-}$

4

$\left[\mathrm{AlF}_{\mathrm{x}}\left(\mathrm{H}_{2} \mathrm{O}\right)_{6-\mathrm{x}}\right]^{(3-x)+}$

$-154.4$

$-155.6$

$-156.0$

$\left[\mathrm{AlF}_{4}\left(\mathrm{H}_{2} \mathrm{O}\right)_{2}\right.$ ]

$\left[\mathrm{AlF}_{3}\left(\mathrm{H}_{2} \mathrm{O}\right)_{3}\right.$ ]

$\left[\mathrm{AlF}_{2}\left(\mathrm{H}_{2} \mathrm{O}\right)_{4}\right]^{+}$

$\left[\mathrm{AIF}\left(\mathrm{H}_{2} \mathrm{O}\right)_{5}\right]^{2+}$

portions of $2.5 \mu \mathrm{L}$ from a $50 \mu \mathrm{L}$ syringe into the fluorescence cuvette. After each step, the fluorescence was monitored for 4 minutes using the same parameters as above.

Titrations with other salt solutions and with the unlabeled MOF were performed accordingly, using $4.8 \mathrm{mM}$ stock solutions of the corresponding salts and a MOF mass concentration of $33 \mu \mathrm{g} / \mathrm{mL}$.

For data analysis, the mean fluorescence signal was calculated for each salt addition step from the last 60 seconds of the 4 minutes measurement interval (typically, the change in fluorescence signal was negligible 10 to 180 seconds after salt addition), the background fluorescence (i.e. the fluorescence signal before fluoride addition) was subtracted, the values were normalized to the resulting starting intensity, and then plotted against the anion concentration in the cuvette. Dilution of the solution due to volume changes caused by the addition of the salt solutions was neglected (the total volume change was $2 \%$ max.). Errors were calculated from the deviation of two individual measurements made with different MOF and salt stock solutions. Lines through the origin were fitted using a linear regression model.

NMR titrations of $\mathrm{NH}_{2}$-MIL-101(Al)-FITC. For ${ }^{19} \mathrm{~F}$ and ${ }^{29} \mathrm{Al} \mathrm{NMR} \mathrm{titrations,} \mathrm{a} \mathrm{stock}$ solution of $\mathrm{NH}_{2}$-MIL-101(Al)-FITC in $\mathrm{H}_{2} \mathrm{O}$ with a mass concentration of $1.2 \mathrm{mg} / \mathrm{mL}$ was prepared. Then, $0,40,80,120,160,200,240,280,320,360$ and $400 \mu \mathrm{L}$ of freshly prepared aqueous $\mathrm{NaF}$ stock solutions $(7.43 \mathrm{mM})$ was added to $400 \mu \mathrm{L}$ of the MOF stock solution in an $1.5 \mathrm{~mL}$ Eppendorf cup, and the total volume was adjusted to $800 \mu \mathrm{L}$ with $\mathrm{H}_{2} \mathrm{O}$. The resulting solutions were mixed by shaking on a vortexer and by sonication in an ultrasonic bath and subsequently transferred to an NMR tube.

Additional information on sample synthesis and characterization as well as on data analysis can be found in the Supplementary Information.

1. McDonagh, C., Burke, C. S. \& MacCraith, B. D. Optical Chemical Sensors. Chem. Rev. 108, 400-422 (2008).

2. Stich, M. I. J., Fischer, L. H. \& Wolfbeis, O. S. Multiple fluorescent chemical sensing and imaging. Chem. Soc. Rev. 39, 3102-3114 (2010).

3. Wolfbeis, O. S. Fiber-Optic Chemical Sensors and Biosensors. Anal. Chem. 76, 3269-3284 (2004).

4. Schäferling, M. The Art of Fluorescence Imaging with Chemical Sensors. Angew. Chem. Int. Ed. 51, 3532-3554 (2012).

5. Wade, C. R., Broomsgrove, A. E. J., Aldridge, S. \& Gabbaï, F. P. Fluoride Ion Complexation and Sensing Using Organoboron Compounds. Chem. Rev. 110, 3958-3984 (2010).

6. Galbraith, E. \& James, T. D. Boron based anion receptors as sensors. Chem. Soc. Rev. 39, 3831-3842 (2010).

7. Kubik, S. Anion recognition in water. Chem. Soc. Rev. 39, 3648-3663 (2010).

8. Jagtap, S., Yenkie, M. K., Labhsetwar, N. \& Rayalu, S. Fluoride in Drinking Water and Defluoridation of Water. Chem. Rev. 112, 2454-2466 (2012).

9. Dusemund, C., Sandanayake, K. R. A. S. \& Shinkai, S. Selective fluoride recognition with ferroceneboronic acid. J. Chem. Soc. Chem. Comm. 333-334 (1995).

10. Wade, C. R. \& Gabbaï, F. P. Colorimetric turn-on sensing of fluoride ions in $\mathrm{H}_{2} \mathrm{O}$ / $\mathrm{CHCl}_{3}$ mixtures by pyridinium boranes. Dalton Trans. $9169-9175$ (2009).

11. Kim, Y. \& Gabbaii, F. P. Cationic Boranes for the Complexation of Fluoride Ions in Water below the 4 ppm Maximum Contaminant Level. J. Am. Chem. Soc. 131, 3363-3369 (2009).

12. Ke, I., Myahkostupov, M., Castellano, F. N. \& Gabbaï, F. P. Stibonium Ions for the Fluorescence Turn-On Sensing of $\mathrm{F}^{-}$in Drinking Water at Parts per Million Concentrations. J. Am. Chem. Soc. 134, 15309-15311 (2012).

13. Descalzo, A. B. et al. A new method for fluoride determination by using fluorophores and dyes anchored onto MCM-41. Chem. Comm. 562-563 (2002).

14. Guha, S. \& Saha, S. Fluoride Ion Sensing by an Anion $-\pi$ Interaction. J. Am. Chem. Soc. 132, 17674-17677 (2010).

15. Boiocchi, M. et al. Nature of Urea-Fluoride Interaction: Incipient and Definitive Proton Transfer. J. Am. Chem. Soc. 126, 16507-16514 (2004).

16. Zhao, H. \& Gabbaï, F. P. A bidentate Lewis acid with a telluronium ion as an anion-binding site. Nat Chem 2, 984-990 (2010).

17. Yaghi, O. M. et al. Reticular synthesis and the design of new materials. Nature 423, 705-714 (2003).

18. Kitagawa, S., Kitaura, R. \& Noro, S.-i. Functional Porous Coordination Polymers. Angew. Chem. Int. Ed. 43, 2334-2375 (2004).
19. Ferey, G. Hybrid porous solids: past, present, future. Chem. Soc. Rev. 37, 191-214 (2008).

20. Li, J.-R., Kuppler, R. J. \& Zhou, H.-C. Selective gas adsorption and separation in metal-organic frameworks. Chem. Soc. Rev. 38, 1477-1504 (2009).

21. Sumida, K. et al. Carbon Dioxide Capture in Metal-Organic Frameworks. Chem. Rev. 112, 724-781 (2011).

22. Ferey, G. et al. Why hybrid porous solids capture greenhouse gases? Chem. Soc. Rev. 40, 550-562 (2011).

23. Allendorf, M. D., Bauer, C. A., Bhakta, R. K. \& Houk, R. J. T. Luminescent metalorganic frameworks. Chem. Soc. Rev. 38, 1330-1352 (2009).

24. Kreno, L. E. et al. Metal-Organic Framework Materials as Chemical Sensors. Chem. Rev. 112, 1105-1125 (2012).

25. Yanai, N. et al. Gas detection by structural variations of fluorescent guest molecules in a flexible porous coordination polymer. Nat Mater 10, 787-793 (2011).

26. Lu, G. et al. Fabrication of Metal-Organic Framework-Containing Silica-Colloidal Crystals for Vapor Sensing. Adv. Mater. 23, 4449-4452 (2011).

27. Stylianou, K. C. et al. A Guest-Responsive Fluorescent 3D Microporous Metal-Organic Framework Derived from a Long-Lifetime Pyrene Core. J. Am. Chem. Soc. 132, 4119-4130 (2010).

28. Takashima, Y. et al. Molecular decoding using luminescence from an entangled porous framework. Nat. Commun. 2, 168 (2011).

29. Serra-Crespo, P., Ramos-Fernandez, E. V., Gascon, J. \& Kapteijn, F. Synthesis and Characterization of an Amino Functionalized MIL-101(Al): Separation and Catalytic Properties. Chem. Mater. 23, 2565-2572 (2011).

30. Imhof, A. et al. Spectroscopy of Fluorescein (FITC) Dyed Colloidal Silica Spheres. J. Phys. Chem. B 103, 1408-1415 (1999).

31. Bojarski, C., Grabowska, J., Kulak, L. \& Kusba, J. Investigations of the excitation energy transport mechanism in donor-acceptor systems. Journal of Fluorescence 1, 183-191 (1991).

32. Agarwal, R. P. \& Moreno, E. C. Stability constants of aluminium fluoride complexes. Talanta 18, 873-880 (1971).

33. Bellack, E. \& Schouboe, P. J. Rapid photometric determination of fluoride with SPADNS-zirconium lake. Anal. Chem. 30, 2032-2034 (1958).

34. Atkins, P. W. Physikalische Chemie. 2 edn, (Wiley-VCH, 1996).

35. World Health Organization (WHO), Fluoride in Drinking-water by Fawell, J., Bailey, K., Chilton, J., Dahi, E., Fewtrell, L. \& Magara, Y. ISBN: 1900222965 , Published by IWA Publishing, London, UK, (2006).

36. American Public Health Association, American Water Works Association, Water Environment Federation, Standard methods for the examination of water and wastewater, American Public Health Association, (1998).

37. Sur, S. K. \& Bryant, R. G. ${ }^{19} \mathrm{~F}$ and ${ }^{27} \mathrm{Al} \mathrm{n.m.r.} \mathrm{spectroscopic} \mathrm{study} \mathrm{of} \mathrm{the} \mathrm{fluoro}$ complexes of aluminum in aqueous solution and in zeolites: Dealumination of zeolites by fluoride ions. Zeolites 16, 118-124 (1996).

38. Bodor, A., Toth, I., Banyai, I., Szabo, Z. \& Hefter, G. T. ${ }^{19}$ F NMR Study of the Equilibria and Dynamics of the Al3 +/F- System. Inorg. Chem. 39, 2530-2537 (2000).

\section{Acknowledgements}

This work was supported by the DFG Priority Program (SPP 1362), the Center for NanoScience (CeNS) and the cluster of excellence Nanosystems Initiative Munich (NIM).

\section{Author contributions}

T.B. supervised the project. F.M.H. and B.R. designed the experiments. B.R. developed the idea for fluoride sensing. F.M.H. performed the synthetic work and B.R. carried out the fluorescence spectroscopy experiments with assistance of S.W. K.K. collected the ${ }^{19} \mathrm{~F}$ and ${ }^{27} \mathrm{Al} \mathrm{NMR}$ data. NMR data analysis was carried out by K.K. and B.R. All authors contributed to writing the manuscript.

\section{Additional information}

Supplementary information accompanies this paper at http://www.nature.com/ scientificreports

Competing financial interests: The authors declare no competing financial interests. 
How to cite this article: Hinterholzinger, F.M., Rühle, B., Wuttke, S., Karaghiosoff, K. \& Bein, T. Highly sensitive and selective fluoride detection in water through fluorophore release from a metal-organic framework. Sci. Rep. 3, 2562; DOI:10.1038/srep02562 (2013). (c) (i) $\odot$ This work is licensed under a Creative Commons AttributionBy No NonCommercial-NoDerivs 3.0 Unported license. To view a copy of this license, visit http://creativecommons.org/licenses/by-nc-nd/3.0 